\title{
Executive Function Deficits in Bipolar Disorder
}

\author{
Weiyi Jiang ${ }^{1, *}, \dagger$, Zili Xu ${ }^{2, *}, \dagger$, Jiuzhang Zhao ${ }^{3, *, \dagger}$ \\ ${ }^{1}$ School of Psychology, University of Washington, Seattle, Washington (98105), USA \\ ${ }^{2}$ School of Psych and Brain Sciences, University of California, Santa Barbara, California (93106), USA \\ ${ }^{3}$ School of Foreign Studies, University of International Relations, Haidian, Beijing 100091, China \\ "Corresponding author.Email:wj29@uw.edu, zilixu@ucsb.edu,20171203@uir.edu.cn \\ tThese authors contributed equally.
}

\begin{abstract}
Executive functions, defined as higher-level cognitive functions, allow lower-level control and regulation to achieve future goals, which are essential for social functioning. Executive function deficits are crucial for understanding bipolar disorder. In the literature, many studies have indicated that patients with bipolar disorder have impairments in theory of mind and social cognition, but there are few direct investigations of executive function deficits. Our study will focus on executive function deficits at different developmental stages in bipolar disorder, related neural mechanisms in bipolar disorder, and the association with social functioning. Executive function deficit in bipolar disorder could happen at different developmental stages of life, and different types of bipolar disorder are accompanied with different neural abnormalities and executive deficits. Moreover, executive function and social cues are closely related. While research about executive function and social functioning is scarce, most of the studies inquire into the correlation between these two, rather than causation. We need furthermore detailed and longitude studies on executive function and social functioning.
\end{abstract}

Keywords: bipolar disorder, social functioning, executive function.

\section{INTRODUCTION}

One's social competence is the coordination of their cognitive processes, which helps them to meet social demands and achieve social goals [1]. It is important for people because poor social competence could lead to psychological distress and reduced self-esteem, which may cause social isolation and a suffered quality of life [2]. Executive functions (EF) are "higher-level" cognitive functions that control and regulate "lower-level" cognitive processes and behaviors that are directed to goal and oriented to future, for example cognitive flexibility, self-control, and time management, which are directed to finishing tasks [3]. EF are important for their effects on our social competence.

Previous studies on the effects of EF on social competence have shown that there are positive associations between EF and social competence. Tremblay discovered that as children interact with society as they grow up, they build up their EF, which make them learn to be less aggressive, thus, their behaviors are more socially acceptable.[1, 4]. The competence resolving peer conflict is indicated associated with working memory (one of the EF), which is the ability to keep the memory of the information and use them [1]. Those with better working memory are more possibly to ensure both their personal interests in the conflict and a positive relationship with the other one involved in the conflict. It is also suggested that EF have made a great contribution to social and emotional functioning [2]. Thus, the study of EF could bring benefit to the study of making better interventions. In this study, the previous studies of the associations between EF and bipolar disorder are going to be reviewed and discussed.

DSM-5 describes bipolar disorders (BDs) as brain disorders that cause extreme fluctuation in individual mood, energy, and functioning [5]. Three different conditions categorize $\mathrm{BDs}$ into the manic-depressive bipolar I disorder with or without psychotic episodes, less severe bipolar II disorder with alternating manic and depressive episodes, cyclic cyclothymic disorder with brief episodes of depression and hypomania. During the manic phase, bipolar disorder (BD) patients show euphoria, irritability, increased energy and activity, inflated self-esteem, excessive talk accompanied by racing thoughts, impulsiveness, and less need for sleep, while during the depressive phase, BD patients show 
depressed mood, low self-esteem, helplessness, fatigue, poor coordination, insomnia or oversleeping, poor concentration, lack of interests in activities, and suicidal attempts. BD patients generally have emotional and social difficulties. Many studies of BDs focus on patients' deficits in social cognition that guide how people process, store and apply information related to other people and the social environment. According to Samamé, BD patients had deficits in theory of mind and emotion recognition in manic, depressed, and euthymic phases, and euthymic patients and subjects at risk for BD tended to have altered face emotion recognition, and brainrelated abnormalities [6]. The results of ToM tasks constantly showed that BD patients suffered largely from mentalizing impairment during both manic and depressed phases and euthymia, and euthymic subjects reported cognitive but intact affective ToM in the Faux pas' task and the Movie for the Assessment of Social Cognition. Many experiments showed that euthymic subjects have abnormal brain functioning, specifically reduced prefrontal activation, and $\mathrm{BD}$ patients tend to have inaccuracy of perceiving and judging emotions. During mania, BD patients tend to misjudge sad expressions, whereas, during depression, positive and negative results are shown along with mood congruent bias (differential reduction in sensitivity to facial expression). Meanwhile, studies showed that manic patients reported impaired performance and selective defects in the recognition of fear and disgust; BD patients tend to perceive fear less accurately. In the aspect of the relationship between social cognition and clinical factors (e.g. age at illness onset and subsyndromal symptomatology), some studies found no significant differences in affect recognition and the theory of mind in both bipolar subgroups, while others found some differences in performance of fear recognition, emotional processing abilities, and the intelligence quotient between $\mathrm{BD}$ I and BD II patients. According to Lahera and colleagues, compared with high-functioning $\mathrm{BD}$ patients, low-functioning BD patients showed a significant impairment in emotion recognition and Faux pas [7].

Social cognition has been studied a lot in BD, previous studies have suggested that there are impairments in theory of mind and emotion recognition. Patients with BD are found with abnormal brain functioning, for instance, reduced prefrontal activation. Executive function will affect an individual's social functioning. Social competence coordinates with an individual's cognitive processes which can help solve peer conflict associated with working memory. Research on executive function in $\mathrm{BD}$ is still limited. Understanding executive function is essential for future development of BD. We will demonstrate studies that are related to this gap and discuss them in 3 aspects: executive function deficits at different developmental stages in $\mathrm{BD}$, related neural mechanisms in $\mathrm{BD}$, and the association with social functioning.

\section{EXECUTIVE FUNCTION DEFICITS IN BD ACROSS DEVELOPMENTAL STAGES}

EF contains cognitive flexibility, inhibition, and so on. One's cognitive flexibility is their ability to adjust their thinking and behavior in order to adapt to the changed rules. Inhibition, or inhibitory control, is the ability to control our attention or behavior (for example inhibit our instinctive reaction) in order to finish tasks. Previous studies have found that compared with healthy people, bipolar patients perform badly regarding EF. In the study by Anabel Martínez-Arán et al., BD patients and a healthy comparison group were asked to complete the tasks testing their executive functions [8]. The tests are the Wisconsin Card Sorting Test (testing cognitive flexibility by changing rules), the Stroop Color and Word Test (testing inhibition for people respond faster to the meaning of the word than color), the Controlled Oral Word Association Test FAS and the animal-naming subtests (testing verbal fluency, which is related to cognitive flexibility and inhibition). The result showed that compared with the healthy subjects, subjects with BD had cognitive dysfunction in verbal fluency and frontal executive tasks. Furthermore, EF would affect BD patients' occupational status. In the study by Julia Drakopoulos et al., partially or fully remitted BD patients were stratified into two groups (an active group and an inactive one) based on their number of working or studying hours per week [9]. They took tests include the Color-Word Interference Test (pointing out the meaning and the color of the word, e.g. "red" with letters in green color. Testing inhibition), the Verbal/Design Fluency Test (listing words from a given category/making different designs by connecting dots, with time limit), the Trail Making Test (match numbers with letters so that the numbers could represent the letters' order. Testing cognitive flexibility), and so on. They also took IQ tests. The result showed that the relevance of the occupational status in BD to EF is more significant than to IQ. These studies have shown that BD has a strong relationship with the deficits of EF, and EF is significantly affecting BD patients' quality of life, thus needs our attention.

It is worth paying attention to $\mathrm{BD}$ in childhood and adolescence, because the plasticity of prefrontal cortex during the early developmental stage means that early intervention is of benefit to the treatment. Moreover, the diagnosis of BD in children and adolescents was growing sharply. From the mid-1990s to the mid-2000s, children's diagnosis rate of BD had been doubled in the US, and the number of youths hospitalized for BD in Germany had increased by $68.5 \%$ [10]. Studies have shown that BD particularly impairs the cognitive flexibility domain of neurocognitive performance. Cognitive flexibility is important to people's life as it helps people to finish different kinds of daily tasks. Hence, children lack of cognitive flexibility could meet more difficulties in school that they cannot adapt to such situation: the 
schedule or the number of the classroom in which they are going to have the class is changed. They could show anxiety because the changed schedule or the classroom is different with the one they have already gotten used to. While those with cognitive flexibility can easily adapt to the change by imagining a new map and a new timetable. In the study by Daniel P. Dickstein et al., children and adolescents with threshold and sub-threshold BD took the test of choosing the correct option in which rules changed few times. The result showed that those with BD-I/II take longer than those with BD-Not Otherwise Specified to complete the reversal task. Therefore, cognitive flexibility is one of the EF which could show deficit in BD in childhood.

EF impaired in old age BD also needs attention, for it is an important cause of disability [11]. Even common people's EF could be impaired while getting old, and the impairment of cognitive function (which includes EF) indicates that it has relation with the high risk of death from ischaemic stroke [12]. Hence, the inconvenience in elder bipolar patients would be more severe for BD would further impair their EF. Old people with impaired EF would quickly forget what they have just heard or read, they could get overly emotional, and they could also face difficulties to organize their thoughts. However, the consistent data about old BD patients is still not enough, and we still do not know much about the elders' cognitive signature of BD. Previous studies have shown that compared with early-onset $\mathrm{BD}$, late-onset $\mathrm{BD}$ would cause severer cognitive disabilities [13-15]. Moreover, studies showed that for elders, cognitive impairment would further cause more impact on their already impaired functions affected by $\mathrm{BD}$ [16]. In order to find out which kinds of EFs are impaired most in old age bipolar depression, Leonardo Caixeta and collages tested old outpatients with early-onset BD by preforming test forms, cards, Stroop test, and verbal fluency test [17]. The result showed that the most impaired EFs are working memory (working memory makes us able to manipulate the information to finish tasks), inhibitory control, mental flexibility, and information processing speed. Besides, in executive assessment, elders' performance could be divided into two kinds: impairment in single EF domain, and in multiple EF domain with or without the syndrome of pseudodementia. Moreover, since some cognitive processes require lots of efforts, the lack of mental energy might explain executive dysfunction. These have shown that for people with BD, their EF would deteriorate after getting old.

\section{EXECUTIVE FUNCTION RELATED NEURAL ABNORMALITIES IN BD}

Executive deficits in BDs refer to cognitive, behavioral, and emotional difficulties that bipolar patients suffer from. The presentation and severity of executive deficits differ in different BD subtypes. In neuroanatomy, the thickness of the cortex is a morphological indicator of the brain, used to describe the total thickness of the cerebral cortex in the mammalian brain. Generally, greater gray matter volume in these related cortical areas is associated with higher IQ scores, so cortical thickness can provide more insights into the relationship between brain structure and intelligence. According to Abe and colleagues, the brain structurefunction relationships are altered in $\mathrm{BD}$, and the corresponding clusters where cortical thickness exists differed in BD subtypes [18]. Thicker cortices of prefrontal brain regions are commonly associated with better executive function performance. Involved in planning complex cognitive behavior, personality expression, decision-making, regulating social behavior, and regulating certain aspects of speech and language, the prefrontal cortex is the cerebral cortex that covers the frontal lobe.

In $\mathrm{BD}$ II patients, executive function performance is positively related with cortical thickness in the bilateral inferior precentral cortex, right medial superior frontal cortex, and lateral prefrontal cortex. Contrarily, in BD I patients, the positive correlations between cognitive performance and cortical thickness are shown in a small part of the left lateral frontal cortex. Moreover, in BD II patients, there are more correlations in the right medial prefrontal regions, compared to those of BD I patients; brain structure-function relationships were found in BD II, not BD I, which is consistent with the previous finding that structural abnormalities exist in BD I, not BD II. The cortical integrity of prefrontal brain regions, frontal cortical thickness, reflected by cortical thickness is positively associated with executive functioning [18]. Studies found that there is abnormal functional connectivity between the medial prefrontal cortex, a part of the default mode network, and other brain areas in BD patients, showing a disengagement between brain areas of the task-positive network and the default mode network in BD. Such structural impairment can potentially lead to only partial recruitment of medial prefrontal networks in BDI, which could explain no correlations observed in medial prefrontal regions in BDI. Similarly, although previous research results showed that BD patients were unable to activate brain regions related to task performance under controlled conditions, based on performance scores of executive functions, BDI patients still manifested better executive function compared to BDII patients [18]. Compared to other subtypes of BD, BDI patients demonstrate unique neural abnormalities related with executive functioning. Previous research showed that Euthymic BDI patients are impaired in verbal memory and executive functioning [19]. According to Liang and colleagues, BDI patients have abnormal DMN temporal dynamics of spontaneous activity, declined PCC-mPFC connection variety and the connection between the medial prefrontal cortex (mPFC) which is the main source of cardiovascular sympathetic 
neurons and involve in numerous sensorimotor, cognitive, and affective processes. The posterior cingulate cortex (PCC) is a part of limbic lobe involving in learning and memory, and it is located posterior to the anterior cingulate cortex and transmits signals from thalamus and neocortex to entorhinal cortex via the cingulum.

Results showed that decreased temporal variabilities are associated with executive dysfunction in BDI patients. Specifically, the reduced PCC-MPFC connectivity relates to the impairment of executive function. Simultaneously, since mPFC is a component of DMN, brain regions exhibit strong low-frequency oscillations in a static state, and are thought to focus on internal mental state processes including mutual inductance, autobiographical memory retrieval or imagining the future. The positive correlation between $\mathrm{mPFC}$ and executive performance may indirectly demonstrate that the dysfunctions of DMN are closely related to emotional and executive impairment of BD I to some degree [19]. Moreover, BD subtypes differ in brain structure-function relationships. BD II patients tend to have lower executive functioning, compared to $\mathrm{BD}$ I patients; the corresponding cluster that demonstrates this phenomenon is detected in the right lateral prefrontal area in the rostral middle frontal cortex. The decreased temporal variability in local region and inter-regional cooperation in BD I is likely to be the neural substrate of executive-function deficits [19].

Functional connectivity is defined as the temporal overlap of neurophysiological events in distant space. If there is a statistical relationship between the activity measures recorded in two regions, the two regions are considered to show functional connectivity. The static functional connectivity is characterized between $\mathrm{mPFC}$ and the regions of DMN. The variability in resting-state functional connectivity can be an indication of the reduced internetwork flexibility in $\mathrm{BD}$ and a correlate of the cognitive impairment in euthymia, the state of living without mood disturbances [20].

Psychiatric patients, including BD patients, show decreased connectivity variability of various brain networks, lowering the speed of exploiting resources to meet changing task demands [20]. Euthymia is the phase without mood disturbance, during which patients experience feelings of cheerfulness and tranquility and show higher resistance to stress [21]. However, studies show that BD patients in euthymia still display cognitive impairments, implying the not neglectable importance of researching and designing the corresponding treatments. According to Nguyen and colleagues, the variability of resting-state functional connectivity is a correlate of cognitive impairment in periods of euthymia, and the functional connectivity can be an indication of reduced internetwork flexibility in BDs. To investigate the dynamics and functional relationship between the executive function and the speed of information processing in patients with BD, Nguyen and colleagues use a high-resolution sequence using the Human Connectome Project to measure neuropsychological indicators of processing speed and executive function. The results of the study show that the dynamic relationship between $\mathrm{mPFC}$ and PCC has altered in participants with $\mathrm{BD}$, so that over time the relationship is less variable and rigid. $\mathrm{BD}$ patients with the most rigid connectivity dynamics between PCC and MPFC have the slowest processing speed and the lowest level of cognitive set-shifting. The degree of connectivity variability, which allows for greater dynamic range, is positively related to the ability to switch between networks, such that the decreased mPFC-PCC connectivity variability is correlated with slower processing speed and the reduced ability to transfer attention from one topic to another. The variability of functional connections at rest can be an indicator of flexibility in the network, which is reduced in BD and can be a correlate of persistent cognitive impairment in the periods during changes in the psychological state of patients.

Conclusively, studies showed different presentations of executive deficits and the corresponding neural abnormalities in BD subtypes. Brain structure-function relationships alter in $\mathrm{BD}$, and the location of the clusters of cortical thickness differs in BD subtypes. Executive dysfunction in BDI patients tends to be associated with decreased temporal variabilities due to the reduced PCCMPFC connectivity. Likewise, rigid functional connectivity is positively correlated with cognitive impairment in the euthymia phase. Therefore, it is necessary to research targeted treatments for different executive deficits in different subtypes of BD based on the investigation of the corresponding neural abnormalities.

\section{THE RELATIONSHIP BETWEEN EXECUTIVE FUNCTION DEFICITS AND SOCIAL FUNCTIONING IN BD}

There are few direct studies about the association between executive function deficits and social function in patients with $\mathrm{BD}$, while the typical relationship between EF deficits and social function has been investigated. Social cognitive skills are implicated with aging, and social cognition involves the ability to understand social agents and interpret other people's intentions and perspectives, which are considered as Theory of Mind. The comparison of true and false belief tasks is often used to assess an individual's ToM. Older adults often underperformed on false belief reasoning in the ToM tests compared with young and middle-aged adults. Cognitive and neuropsychology models of ToM suggest executive functioning and social cue detection are the two processes that affect age differences on belief perception [22]. A biological motion task in a lifespan 
aging sample was designed to test the ability to decode social cues, and results showed that social cue decoding mediated the age differences in reasoning belief performance. The young and middle-aged groups performed better than the older group in this task. Executive functions are measured by an individual's cognitive control functions which involve updating and inhibition [23-27]. The Results showed that the older adults group was impaired in EF. EF, especially updating in working memory, is also a mediator of age differences. The age difference is a determining factor in decoding social cues, and older adults are impaired in EF, which indicates that EF deficits lead to poor social cue decoding. Therefore, understanding EF is crucial for decoding social cues.

One of the few studies about social function and EF in $\mathrm{BD}$ is the investigation of affective lability. Affective lability is a term that is utilized when it comes to mood disorders where individuals have intense and frequent fluctuations that impact both unpleasant and pleasant events. Affective lability is very important to social function. Uncontrollable mood shifting will affect both daily life and social life, for instance, patients with affective lability might have inappropriate or exaggerated emotional reactions. A previous study has suggested that emotion processing could be affected by cognitive dysfunctions [28], which is referred to as an aspect of executive function [29]. According to Aminoff [30], affective lability is assessed by using Affective Lability Scale (ALS) [31], and patients with BD have higher ALS scores compared with the healthy control group. Executive function is measured by phonetic verbal fluency, semantic verbal fluency, and semantic set shift. Results show that there is a significant positive correlation between affective lability and semantic set shift. While there is no significant association found between impaired cognitive control and affective lability in BD. In general, the higher amount of affective lability in comparison with controls is the characteristic and state-dependent in BD. Besides, the disturbing affective lability emerges from an aberrant connection between cognitive as well as emotional brain networks.

BD during mood episodes as well as in euthymia while cognitive functioning is affected at the same time. Facial Emotional Recognition (FER) is regarded as a key skill to the success of social function [22]. When it comes to social situations facial emotions are significant as they tell individuals the character would react to a specific situation. Facial expression is known as an example from social cues, while EF deficits will affect the ability to interpret social cue decoding. Cognitive deficits and challenges identifying facial emotions are apparent in all mood episodes in BD 1 patients as well as in remission. Even though FER is not regarded as a cognitive area, the findings show that together with $E F$, it plays a complementary function. In the study of David [32], EF is measured by using the Wisconsin Card Sorting Test
[33], and FER is assessed by Pictures of Facial Affect [34]. Bipolar patients have less FER performance than controls on fear subtests, surprise tests, FER total scores, and happiness. Besides, Bipolar 1 maniac patients are demonstrated poorer performance for EF in comparison to the control group. A correlation has been found between EF and FER, in general, people with more integral EF tend to have a better ability to recognize facial expressions.

$\mathrm{EF}$ and social functions are closely related in $\mathrm{BD}$. Impairment in EF can cause poor social cues decoding. On the other hand, EF deficits also affect emotion processing, which is vital for social function. BD patients always have high affective lability scores. Since EF influences social cues decoding, and facial expression is a social cue, EF deficits will weaken the ability to facial emotion recognition.

\section{LIMITATION AND FUTURE DIRECTION}

Executive function is positively associated with social competence that is the coordination of cognitive processes [35-37]. According to previous research, BDs can directly negatively impact patients' living status, job status, and social ability by causing considerable damage to cognitive abilities and cognitive flexibility. Such cognitive dysfunction can lead to social incompetence and executive deficits, which has a considerable social impact and needs social attention for focused research. However, although there are many studies about BDs, relevant studies about executive deficits and social cognition still have been insufficient. Moreover, these few studies about the relationship between executive deficits and social cognition in BD mainly investigate the correlational relationship, not the causal relationship [3840]. The corresponding experimental designs do not fit to conduct causal interpretations. Simultaneously, these studies are mainly limited to the horizontal comparison between normal people and BD patients, and there are too few corresponding longitudinal studies.

Besides the finding of the state-dependent affective lability in $\mathrm{BD}$ due to the abnormal connection between cognitive and emotional brain networks, facial emotion recognition is a critical ability in social intercourse. Studies show a positive correlation between the ability of recognizing facial emotions and the cognitive deficits in BDs and how facial emotion recognition, not a cognitive area but together with executive function, plays a complementary function [40]. Such coexistence of inability to recognize facial expressions and executive deficits in BDs still requires future research to investigate the corresponding neurological basis with a larger sample size. In the future, more detailed and in-depth research on social dysfunction in BDs is needed. 


\section{CONCLUSION}

$\mathrm{EF}$ could be impaired by $\mathrm{BD}$, and this impairment could affect patients' quality of life such as their occupational status, and the EF deficit could happen in different periods of life. Children with BD would show cognitive flexibility deficit, which makes it difficult for them to adapt to changes in their schedules.. The most impaired domains of $\mathrm{EF}$ in old patients are working memory, information processing speed, inhibitory control, and mental flexibility. As for nerves, different subtypes of BD show different neural abnormalities and different executive deficits. The difference also suggests the alteration of brain structure-function relationships and the differentiation of the location of cortical thickness clusters. The reduction of PCC-MPFC connectivity tends to decrease temporal variabilities of BDI patients' executive dysfunction. There is likewise a positive correlation between rigid functional connectivity and cognitive impairment during euthymia. Thus, different subtypes of $\mathrm{BD}$ require different targeted treatments which are based on the study of their corresponding neural abnormalities. In respect of social functioning, EF could also hinder social cues decoding and emotion processing, which are important for social function. Patents' effect is always more labile. EF deficit also weakens the facial emotion recognition ability, because social cues decoding is influenced by EF, and facial expression is one of the social cues. Few previous studies had studied the relations between EF and society, and they had mainly studied their correlation rather than their causation, meanwhile, they merely compared the items horizontally without longitudinal study. Therefore, future studies should lay emphasis on the relationship between EF and society.

\section{REFERENCES}

[1] Caporaso, J. S., Boseovski, J. J., \& Marcovitch, S. (2019). The individual contributions of three executive function components to preschool social competence. Infant and Child Development, 28(4), e2132.

[2] Alduncin, N., Huffman, L. C., Feldman, H. M., \& Loe, I. M. (2014). Executive function is associated with social competence in preschool-aged children born preterm or full term. Early human development, 90(6), 299-306.

[3] Alvarez, J. A., \& Emory, E. (2006). Executive function and the frontal lobes: a meta-analytic review. Neuropsychology review, 16(1), 17-42.

[4] Tremblay, R. E. (2010). Developmental origins of disruptive behaviour problems: the 'original sin'hypothesis, epigenetics and their consequences for prevention. Journal of child psychology and psychiatry, 51(4), 341-367.
[5] Jessica Truschel, \& Medically reviewed by: Henry A. Montero. (2020, September 29). Bipolar Definition and DSM-5 Diagnostic Criteria. Psycom.net - Mental Health Treatment Resource Since 1996.

[6] Samamé, C. (2013). Social cognition throughout the three phases of bipolar disorder: A state-of-the-art overview. Psychiatry Research, 210(3), 1275-1286.

[7] Lahera, G., Ruiz-Murugarren, S., Iglesias, P., RuizBennasar, C., Herrería, E., Montes, J. M., \& Fernández-Liria, A. (2012). Social cognition and global functioning in bipolar disorder. Journal of Nervous and Mental Disease, 200(2), 135-141.

[8] Martínez-Arán, A., Vieta, E., Reinares, M., Colom, F., Torrent, C., Sánchez-Moreno, J., ... \& Salamero, M. (2004). Cognitive function across manic or hypomanic, depressed, and euthymic states in bipolar disorder. American Journal of Psychiatry, 161(2), 262-270.

[9] Drakopoulos, J., Sparding, T., Clements, C., Pålsson, E., \& Landén, M. (2020). Executive functioning but not IQ or illness severity predicts occupational status in bipolar disorder. International journal of bipolar disorders, 8(1), 7 .

[10]Dickstein, D. P., Axelson, D., Weissman, A. B., Yen, S., Hunt, J. I., Goldstein, B. I., ... \& Keller, M. B. (2016). Cognitive flexibility and performance in children and adolescents with threshold and subthreshold bipolar disorder. European child \& adolescent psychiatry, 25(6), 625-638.

[11] Sajatovic, M., Strejilevich, S. A., Gildengers, A. G., Dols, A., Al Jurdi, R. K., Forester, B. P., ... \& Shulman, K. I. (2015). A report on older-age bipolar disorder from the International Society for Bipolar Disorders Task Force. Bipolar disorders, 17(7), 689704.

[12] Gale, C. R., Martyn, C. N., \& Cooper, C. (1996). Cognitive impairment and mortality in a cohort of elderly people. Bmj, 312(7031), 608-611.

[13] Muris, P., Meesters, C., Morren, M., \& Moorman, L. (2004). Anger and hostility in adolescents: Relationships with self-reported attachment style and perceived parental rearing styles. Journal of Psychosomatic Research, 57(3), 257-264.

[14] Schouws, S. N., Comijs, H. C., Stek, M. L., Dekker, J., Oostervink, F., Naarding, P., ... \& Beekman, A. T. (2009). Cognitive impairment in early and late bipolar disorder. The American Journal of Geriatric Psychiatry, 17(6), 508-515.

[15] Schouws, S. N. T. M., Zoeteman, J. B., Comijs, H. C., Stek, M. L., \& Beekman, A. T. F. (2007). 
Cognitive functioning in elderly patients with early onset bipolar disorder. International Journal of Geriatric Psychiatry: A journal of the psychiatry of late life and allied sciences, 22(9), 856-861.

[16] Rise, I. V., Haro, J. M., \& Gjervan, B. (2016). Clinical features, comorbidity, and cognitive impairment in elderly bipolar patients. Neuropsychiatric disease and treatment, 12, 1203.

[17] Caixeta, L., Soares, V. L., Vieira, R. T., Soares, C. D., Caixeta, V., Ferreira, S. B., \& Aversi-Ferreira, T. A. (2017). Executive function is selectively impaired in old age bipolar depression. Frontiers in psychology, 8, 194.

[18] Abé, C., Rolstad, S., Petrovic, P., Ekman, C. -., Sparding, T., Ingvar, M., \& Landén, M. (2018). Bipolar disorder type I and II show distinct relationships between cortical thickness and executive function. Acta Psychiatrica Scandinavica, 138(4), 325-335.

[19] Liang, Y., Jiang, X., Zhu, W., Shen, Y., Xue, F., Li, Y., \& Chen, Z. (2020). Disturbances of dynamic function in patients with bipolar disorder I and its relationship with executive-function deficit. Frontiers in Psychiatry, 11.

[20] Nguyen, T. T., Kovacevic, S., Dev, S. I., Lu, K., Liu, T. T., \& Eyler, L. T. (2017). Dynamic functional connectivity in bipolar disorder is associated with executive function and processing speed: A preliminary study. Neuropsychology, 31(1), 73-83.

[21] Purse, M. (2020, September 19). What Is Euthymic Mood in Bipolar Disorder? Verywell Mind.

[22] Phillips, L. H., Bull, R., Allen, R., Insch, P., Burr, K., \& Ogg, W. (2011). Lifespan aging and belief reasoning: Influences of executive function and social cue decoding. Cognition, 120(2), 236-247.

[23] Apperly, I. A., Samson, D., Chiavarino, C., \& Humphreys, G. W. (2004). Frontal and temporoparietal lobe contributions to theory of mind: Neuropsychological evidence from a false-belief task with reduced language and executive demands. Journal of Cognitive Neuroscience, 16(10), 17731784.

[24] Frith, U., \& Frith, C. (2010). The social brain: Allowing humans to boldly go where no other species has been. Philosophical Transactions of the Royal Society B: Biological Sciences, 365(1537), 165-176.

[25] Gallagher, H., Happé, F., Brunswick, N., Fletcher, P., Frith, U., \& Frith, C. (2000). Reading the mind in cartoons and stories: An fMRI study of 'theory of mind' in verbal and nonverbal tasks. Neuropsychologia, 38(1), 11-21.

[26] Saxe, R., Carey, S., \& Kanwisher, N. (2004). Understanding other minds: Linking developmental psychology and functional neuroimaging. Annual Review of Psychology, 55(1), 87-124.

[27] Singer, T. (2006). The neuronal basis and ontogeny of empathy and mind reading: Review of literature and implications for future research. Neuroscience \& Biobehavioral Reviews, 30(6), 855-863.

[28]Phillips, M. L., Drevets, W. C., Rauch, S. L., \& Lane, R. (2003). Neurobiology of emotion perception II: Implications for major psychiatric disorders. Biological Psychiatry, 54(5), 515-528.

[29] Melcher, T., Falkai, P., \& Gruber, O. (2008). Functional brain abnormalities in psychiatric disorders: Neural mechanisms to detect and resolve cognitive conflict and interference. Brain Research Reviews, 59(1), 96-124.

[30] Aminoff, S. R., Jensen, J., Lagerberg, T. V., Hellvin, T., Sundet, K., Andreassen, O. A., \& Melle, I. (2012). An association between affective lability and executive functioning in bipolar disorder. Psychiatry Research, 198(1), 58-61.

[31] Harvey, P. D., Greenberg, B. R., \& Serper, M. R. (1989). The affective lability scales: Development, reliability, and validity. Journal of Clinical Psychology, 45(5), 786-793.

[32] David, D. P., Soeiro-de-Souza, M. G., Moreno, R. A., \& Bio, D. S. (2014). Facial emotion recognition and its correlation with executive functions in bipolar I patients and healthy controls. Journal of Affective Disorders, 152-154, 288-294.

[33] Lezak, M. D. (1995). Neuropsychological assessment. Oxford University Press.

[34] Ekman, P., \& Friesen, W. V. (1976). Pictures of facial affect.

[35] Julie A. Alvarez \& Eugene Emory. (2006). Executive Function and the Frontal Lobes: A MetaAnalytic Review. Neuropsychology Review, Vol. 16, No. 1

[36] Jessica S. Caporaso, Janet J. Boseovski, \& Stuart Marcovitch. (2019). The individual contributions of three executive function components to preschool social competence. Inf Child Dev. 2019;e2132

[37] Nidia Alduncin, Lynne C. Huffman, Heidi M. Feldman, \& Irene M. Loe. (2014). Executive Function is Associated with Social Competence in Preschool-Aged Children Born Preterm or Full Term. Early Hum Dev. 2014 June ; 90(6): 299-306. 
[38] Phillips, L. H., Bull, R., Allen, R., Insch, P., Burr, K., \& Ogg, W. (2011). Lifespan aging and belief reasoning: Influences of executive function and social cue decoding. Cognition, 120(2), 236-247.

[39] Aminoff, S. R., Jensen, J., Lagerberg, T. V., Hellvin, T., Sundet, K., Andreassen, O. A., \& Melle, I. (2012). An association between affective lability and executive functioning in bipolar disorder. Psychiatry Research, 198(1), 58-61.

[40] David, D. P., Soeiro-de-Souza, M. G., Moreno, R. A., \& Bio, D. S. (2014). Facial emotion recognition and its correlation with executive functions in bipolar I patients and healthy controls. Journal of Affective Disorders, 152-154, 288-294. 